1 Prevalence of $C 9 O R F 72$ expansion in a large series of patients with idiopathic \title{
normal pressure hydrocephalus
}

Korhonen Ville E, BMed ${ }^{a^{*}}$, Remes Anne M, MD, PhD ${ }^{\mathrm{b}, \mathrm{c}, \mathrm{de}}$, Helisalmi Seppo, MSc, $\mathrm{PhD}^{\mathrm{b}}$, Rauramaa Tuomas, $\mathrm{MD}, \mathrm{PhD}^{\mathrm{f}}$,Sutela Anna, $\mathrm{MD}, \mathrm{PhD}^{\mathrm{g}}$, Vanninen Ritva, $\mathrm{MD}, \mathrm{PhD}^{\mathrm{g}}$, Suhonen NooraMaria, MSc, $\mathrm{PhD}^{\mathrm{c}, \mathrm{e}}$,Haapasalo Annakaisa $\mathrm{MSc} \mathrm{PhD}^{\mathrm{h}}$, Hiltunen Mikko MSc, $\mathrm{PhD}^{\mathrm{i}}$, Jääskeläi nen Juha E, MD, $\mathrm{PhD}^{\mathrm{a}}$, Soininen Hilkka MD, $\mathrm{PhD}^{\mathrm{b}}$, Koivisto Anne $\mathrm{M}$,

$\mathrm{MD}, \mathrm{PhD}^{\mathrm{b}, \mathrm{d}}$ and Leinonen Ville, $\mathrm{MD}, \mathrm{PhD}^{\mathrm{a} *}$

${ }^{a}$ Department of Neurosurgery, Kuopio University Hospital and University of Eastern Finland, Kuopio, Finland

${ }^{\mathrm{b}}$ Institute of Clinical Medicine - Neurology, University of Eastern Finland, Kuopio, Finland.

${ }^{\mathrm{c}}$ Medical Research Center, Oulu University Hospital, Oulu, Finland

${ }^{\mathrm{d}}$ Department of Neurology, Kuopio University Hospital, Kuopio, Finland

${ }^{\mathrm{e}}$ Unit of Clinical Neuroscience, Neurology, University of Oulu, Oulu, Finland

${ }_{\mathrm{f}}^{\mathrm{f}}$ Institute of Clinical Medicine - Pathology, School of Medicine, University of Eastern Finland and Department of Pathology, Kuopio University Hospital, Kuopio, Finland.

${ }^{\mathrm{g}}$ Department of Radiology, Kuopio University Hospital and University of Eastern Finland, Kuopio, Finland

${ }^{\text {h} A . I . ~ V i r t a n e n ~ I n s t i t u t e ~ f o r ~ M o l e c u l a r ~ S c i e n c e s, ~ U n i v e r s i t y ~ o f ~ E a s t e r n ~ F i n l a n d, ~ K u o p i o, ~ F i n l a n d ~}$

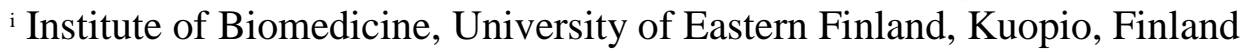

*Corresponding author

Ville Korhonen, BMed, PhD student

Department of Neurosurgery

Kuopio University Hospital

P.O. Box 1777, FI-70211 Kuopio, Finland

Tel: +358-40-514 7320; Fax: +358-44-717-2303

E-Mail: villekor@uef.fi

http://www.uef.fi/nph

Running title: C9ORF72 and idiopathic normal pressure hydrocephalus

Keywords: C9ORF72, FTLD, BvFTD, Idiopathic Normal Pressure Hydrocephalus, Dementia

Conflicts of interest: Nothing to disclose

Funding: The study was supported by research grants from the Academy of Finland, the Kuopio University Hospital VTR Fund (5252614), Sigrid Juselius Foundation, the Finnish Medical Foundation, Olvi Foundation, Kuopio University Hospital Research Foundation, Emil Aaltonen Foundation, and the Finnish cultural foundation - North-Savo regional Fund.

Acknowledgements: We wish to acknowledge BLS Marjo Laitinen for her expert assistance with the laboratory analysis, RN Marita Parviainen for her kind technical assistance, Anna M. Järvinen, $\mathrm{PhD}$, for editing the English language, Tiina Suominen, $\mathrm{PhD}$ (University of Tampere) and prof. Bjarne Udd (University of Tampere) for verifying the genotyping results. 
51 Prevalence of the C9ORF72 expansion in a large series of patients with 52 idiopathic normal pressure hydrocephalus

53

54

55

56

57

58

59

60

61

62

63

64

65

66

67

68

69

70

71

72

73

74

75

76

78

79

80

81 77

\section{Abstract:}

Background/Aims: The C9ORF72 expansion is known to cause frontotemporal lobar degeneration (FTLD) and amyotrophic lateral sclerosis (ALS). We aim to identify the prevalence of the C9ORF72 expansion in idiopathic normal pressure hydrocephalus (iNPH) Methods: We analysed the C9ORF72 expansion in a large cohort of patients with possible iNPH $(\mathrm{n}=487)$ and cognitively intact elderly controls ( $n=432$; age $>65$ years). Results: While the C9ORF72 expansion was detected in $1.6 \%(n=8 / 487)$ of cases with possible iNPH, no control subject was found to carry the mutation. The mean age at onset of symptoms of C9ORF72 expansion carriers was 59 years (range: 52-67 years), 11 years less than non-carriers ( $\mathrm{p}=0.0002)$. The most frequent initial/main symptom pertained to gait difficulties. Despite identified mutation, only three of the patients fulfilled the criteria for FTLD-ALS spectrum. Clinically significant shunt response was detected in 6 out of 7 shunted C9ORF72 expansion carriers. Conclusion: This is the first study cohort identifying the underlying C9ORF72 expansion in patients with iNPH providing evidence for the potential comorbidity between iNPH and FTLD-ALS spectrum. Analysis of the C9ORF72 expansion should be considered for patients with probable iNPH presenting with frontal atrophy and personality changes or other severe psychiatric symptoms.

Running title: Prevalence of C9ORF72 expansion in iNPH 1 (3) 4 75 6 78 79 80 81 


\section{Introduction}

Idiopathic normal pressure hydrocephalus (iNPH) and frontotemporal lobar degeneration (FTLD) are cognitive disorders presenting with overlapping clinical features. INPH is characterized by a triad of core symptoms, namely gait difficulties, cognitive impairment, and urinary incontinence.

The symptoms are progressive in nature, and patients may express the triad only partially or fully $(1,2)$. In iNPH, ventricular enlargement comprises a key radiological finding alongside the observable characteristic clinical symptoms $(3,4)$ and the observed cognitive impairment is usually characterized by psychomotor slowness, impairment in frontal executive functions, as well as other symptoms reflecting frontal-subcortical dysfunction $(1,4,5)$. By contrast, FTLD is a clinically heterogeneous group of syndromes that includes behavioural variant frontotemporal dementia (bvFTD), semantic variant primary progressive aphasia (svPPA), and non-fluent variant primary progressive aphasia (nfvPPA). BvFTD is the most common clinical subtype of FTLD, which presents with progressive changes in personality and behaviour, together with deficits in executive functions (6). Hexanucleotide repeat expansion in the $C 9 O R F 72$ is the most common genetic cause of bvFTD $(7,8)$. Although amyotrophic lateral sclerosis (ALS) represents the most frequent motor symptom in patients with the C9ORF72 expansion, extrapyramidal symptoms have also been documented $(9,10)$.

On the basis of the similarity of clinical symptoms in bvFTD and iNPH, it was hypothesized that bvFTD may demonstrate comorbidity with iNPH. Importantly, we have previously documented a bvFTD case suffering from comorbid iNPH (11). To this end, the aim of the study was to evaluate the role of the C9ORF72 expansion in a large cohort of iNPH patients. This is the first study addressing systematically the prevalence of the C9ORF72 expansion in iNPH.

\section{Methods}


All experimental procedures complied with the standards of the Declaration of Helsinki and further, the Kuopio University Hospital Research Ethics Board approved the study. All patients provided informed consent. The clinical cohort comprised of 487 patients with possible iNPH (Fig. 1; Table 1). The control group consisted of 432 subjects (mean age at evaluation $72 \pm 3.4$; range 66-87; 159 male), who underwent a thorough evaluation by an experienced neurologist specialized in memory disorders, to rule out neurodegenerative diseases.

INPH diagnosis was carried out by neurologist in accordance with the Relkin et al. (2005)(1)

criteria. The diagnosis of bvFTD was based on the Rascovsky et al. (2011) (6) criteria. Patients who simultaneously presented with at least one of the three core symptoms associated with iNPH, together with enlarged ventricles disproportionate to the size of the sulci of the cerebral convexities (Evan's index >0.30) (14) based on CT or MRI scans, were referred to a neurosurgeon.

Subsequently, a 24-hour intraventricular pressure (IVP) monitoring, spinal tap, or lumbar infusion test was performed by a neurosurgeon.

A ventriculoperitoneal shunt was placed in 409 patients, out of whom $44 \%(n=181)$ were shunted on the basis of the results of the 24-hour IVP monitoring. The remaining patients were shunted according to the following protocol: $32 \%(\mathrm{n}=132)$ of patients were shunted based on a positive taptest (at least $20 \%$ increase in walking speed in a 20-meter test, including one turn); $16 \%(\mathrm{n}=66)$ based on a pathological finding (conductance $\leq 10)$ in a lumbar infusion test; and $7 \%(\mathrm{n}=30)$ based on the combination of clinical evaluation and radiological findings.

Shunt response was defined as an improvement in at least two of the classical triad of symptoms, or improvement in one classical symptom accompanied with a significant improvement in headaches, 
131

132

133

134

135

136

137

138

139

140

141

142

143

vertigo, and/or balance. Additionally, objective shunt response evaluation, where pre and post-shunt gait speed was used as a determining variable, was available for some of the patients. Gait speed improvement over $20 \%$ was considered significant (Table 2).

The patients' cognitive functioning was evaluated by the Consortium to Establish a Registry for Alzheimer's Disease Neuropsychological Battery (CERAD-NB) or MMSE. Behavioral symptoms were evaluated by Frontal Behavioural Inventory (FBI). Additionally, extensive follow-up data and medical records were carefully re-evaluated.

The patients' first-degree family history was carefully mapped and history of iNPH, ALS, dementia, or psychiatric diseases were evaluated (reported in table 2).

Brain biopsy was extracted from 425 patients from the right frontal cortex during the insertion of the ventricular catheter for shunt or IVP monitoring. One biopsy was excluded from the cohort due to technical problem (\#6). A neuropathologist evaluated immunohistochemistry for beta-amyloid (6F/3D), hyperphosphorylated tau (AT8), p62, and TDP-43.

Previous MRI (1.5T or 3T) $(\mathrm{n}=6)$ or CT-scans $(\mathrm{n}=2)$ of all C9ORF72 expansion carriers were reevaluated by an experienced neuroradiologists, in order to both characterize and quantify the apparent morphological changes of the brain described in Tables 2 and 3. 
The presence of the C9ORF72 repeat expansion was detected utilizing the Repeat-Primed PCR (RP-PCR) (8). The results of the RP-PCR were confirmed using the Amplicon length analysis (12), and verified in a collaborating laboratory, where the analysis was repeated in a blinded manner.

All of the identified living cases with the C9ORF72 repeat expansion were clinically re-evaluated by an experienced neurologist (AMR) specialized in FTLD (patient \#3 deceased during the followup period).

\section{Results}

The C9ORF72 expansion was detected in 1.6\% (8/487) of cases with possible iNPH. Seven of them fulfilled the clinical diagnostic criteria for probable iNPH, who subsequently underwent the shunting procedure. (Table 2). Additionally, one identified iNPH case with the C9ORF72 expansion failed to fully fulfil the diagnostic criteria for probable iNPH, thus having finally an unlikely iNPH status. The patient in question suffered from severe and widespread cognitive impairment and was diagnosed with both clinically and neuropathologically (\#3) confirmed Alzheimer's disease (AD); thus, ventriculoperitoneal shunt was not placed in this case. One shunted patient was retrospectively diagnosed to be suffering from neuropathologically confirmed FTLD (\#7). None of the control subjects was found to carry the $C 9 O R F 72$ expansion.

The mean onset age of symptoms in patients with the C9ORF72 expansion was significantly lower compared to non-carriers (59 vs 70 years, $95 \%$ CI 5.1-16.7, $p=0.0002$ ). The mean Evan's Index was 0.43 . Disproportion between the sylvian and suprasylvian subarachnoid spaces was present in all but one (\#8) of the patients, with the results ranging from mild (\#2,\#3,\#4, \#6) to severe (\#1, \#5, \#7) (Table 3, Fig 2). 
Six patients were found to present with the full classical triad of symptoms associated with iNPH together with the enlarged ventricles as indicated by brain imaging. In five out of the eight patients, gait difficulty was the leading symptom. The mean pre-operative MMSE score of the sample was 20.8/30 [14-25, median 22]. Results from the CERAD-NB overall showed that five patients were found to exhibit mild to moderate cognitive impairment (\#1, \#4, \#6, \#7 and \#8), with one remaining patient demonstrating unimpaired cognition (\#2), and the other remaining patient showing profound deficits due to severe dementia (\#5) (Table 4). Moreover, two patients suffered from mild depression. The mean follow-up time was 7.4 years.

Two patients demonstrated mild behavioral changes, and one patient displaying moderate deterioration of behaviour. Out of the eight patients, only two cases fulfilled the clinical diagnostic criteria for bvFTD (6), with one of the remaining patients being diagnosed with ALS based on ENMG (\#1). All patients had a family history of symptoms linked to iNPH, ALS, dementia, or psychiatric diseases (Table 2).

Positive clinically verified or objectively measured shunt response was detected in almost all cases when evaluated during multiple follow-ups. (Table 2). The most frequent benefit attributable to the shunt pertained to improvement in gait followed by improvement in urinary incontinence. Some of the patients showed a modest improvement in both episodic memory and executive functions (\#1, \#6, \#7) even though no clinically relevant shunt response was evident in their cognitive abilities. The patient's cognitive abilities showed a tendency to deteriorate over the mean cognitive follow-up period of 10.6 (range 4-70 months) years but some of the patients remained cognitively stable (Table 4).

Beta-amyloid reactivity was common but only one patient presented with both beta-amyloid and tau protein concomitantly (\#3), with the patient subsequently being diagnosed with $\mathrm{AD}$. All of the patients testing positive for beta-amyloid were ApoE4 carriers. P62 and TDP-43 immunohistochemistry were frequently detected but not in all cases (Table 2, Figure 3). 
This is the first study addressing the presence of the C9ORF72 repeat expansion in an iNPH cohort. Surprisingly, the C9ORF72 expansion was found in $1.6 \%$ of patients primarily diagnosed with possible iNPH. Almost all of the C9ORF72 expansion carriers presented with gait difficulties. Recently it has been suggested that the full or intermediate (20-30 repeats) C9ORF72 expansion could be associated with Parkinson's disease (PD) (13) but the results were not replicated in autopsy confirmed study setting or meta-analysis of published research $(14,15)$. However,

The differential diagnostics in iNPH is based on clinical and radiological findings. Response to shunt plays a significant role in confirming the diagnosis, which causes most likely both missed and false diagnoses $(4,21)$. It should be noted that ventriculomegaly can be an early sign of neurodegeneration even when parasagittal sulci are obliterated. In the current study, all shunted patients fulfilled the criteria for probable iNPH but only three of the $C 9 O R F 72$ expansion carriers were determined to additionally suffer from comorbid bvFTD or ALS. It could be that the clinical spectrum of C9ORF72 expansion is variable and the current clinical criteria for bvFTD might not be sensitive enough to identify atypical bvFTD patients $(6,22)$. However, interestingly $6 / 7$ of the shunted $C 9 O R F 72$ expansion carriers in the study cohort were observed to demonstrate a clinically verified shunt response, suggesting that the presence of the C9ORF72 expansion did not hamper the shunt response. However, the shunt surgery failed to significantly impact upon the patients cognitive functioning and the shunt response showed a tendency toward diminishing during the 
follow-up period, with the average duration of 7.4 years. These findings are in line with previous reports on the shunt response $(23,24)(15,16,17,18)$.

Neuropathological evaluation of the frontal cortical biopsies was available for seven of the cases carrying the C9ORF72 expansion. Unfortunately, neuropathological analyzes were not systematically performed for p62 and TDP-43 pathology thereby diminishing both the validity and the generalizability of the current results. The C9ORF72 expansion has been associated with TDP43 pathology with or without p62 immunoreactive inclusions (27). In the current cohort, p62 immunoreactive inclusions were detected more frequently as compared to TDP-43 pathology, which may either be due to the small size of the frontal cortical biopsy (1 $\mathrm{mm}$ x 3-5 $\mathrm{mm}$ in size), and/or some phenomena associated with the C9ORF72 expansion. It has previously been shown that the presence of ubiquitin together with p62 positive and TDP-43 negative neuronal inclusions, especially in the cerebellum, hippocampus and thalamus, are highly specific to the C9ORF72 expansion $(27,28)$.

In the current study, AD-related neuropathology characterized by beta-amyloid plaques without taupathology was observed in two cases. Each of the beta-amyloid positive cases was an ApoE4 allele carrier. It has been previously reported that AD-associated neuropathology is occasionally linked to the C9ORF72-associated FTLD (29). In addition, similarly to the AD patients, abnormally low CSF $\mathrm{A} \beta_{1-42}$ levels have been identified in over $20 \%$ of the FTLD patients with the C9ORF72 expansion (30). As AD and vascular dementia are the most frequent cognitive disorders showing comorbidity with iNPH, the pathologic features perceived in iNPH most commonly represent both vascular and AD-related changes (31). Thus, the current results are consistent with the previous evidence of typical TPD-43- and p62-positive inclusions in patients with the C9ORF72 expansion. Furthermore, the present findings provide supporting evidence to the assumption that mixed neuropathology may be present both in cases with the C9ORF72 expansion, as well as in iNPH patients. 
Differential diagnosis between iNPH and bvFTD can be challenging due to the overlapping clinical and imaging features. For example, psychomotor slowness and deficits in executive functions represent typical features of both diseases $(1,4-6)$. Difficulties with gait and balance form the core symptomatology of iNPH (1), and bvFTD patients also may suffer from extrapyramidal symptoms, such as gait impairment, bradykinesia, urinary incontinence, and tremor. Moreover, psychiatric symptoms are commonplace in bvFTD patients resulting in erroneous diagnoses of psychiatric disease at the beginning of the disease course (6). It is possible that also iNPH is associated with a constellation of psychiatric manifestations, including aggression, disturbances of impulse control, and even psychosis $(1,3)$. Furthermore, Schizophrenia is an over-represented comorbidity in iNPH (32). The diagnostic process is further complicated by the fact that neuroradiological differential diagnostics are not clear in all cases. Ventricular enlargement is a key radiological finding in iNPH (1), and may also be present in the C9ORF72 expansion-associated bvFTD $(9,33)$.

Despite the growing evidence, the etiology of iNPH remains elusive. However, accruing literature suggests that iNPH likely is associated with a genetic component (34-39). The current findings showing that the C9ORF72 expansion is associated with the iNPH phenotype in a subset of patients, appears to represent a further genetic variation with potential linkages to iNPH. Normal ventricular wall - ependyma - is covered by motile cilia and primary ciliary dyskinesias have been linked with congenital hydrocephalus (40). The C9ORF72 expansion could, through yet unknown mechanisms, relate to ependymal dysfunction or the impaired clearance of harmful metabolites and therefore advance the progression of iNPH.

The main strengths of the current study include the large cohort of iNPH patients, which provides a unique opportunity to capture even the rather sporadic potential comorbid features that may be shared between iNPH and other neurodegenerative disorders. Secondly, the assessment of the shunt response combined with the evaluation of clinical characteristics of iNPH allows for reliable diagnosis of probable iNPH. Moreover, brain biopsies from the frontal cortex offer a rare albeit a 
powerful window into the neuropathological changes in the living brain tissue. The limitations include a potential for type one error due to the low frequency of the $C 9 O R F 72$ expansion and the absence of the C9ORF72 expansion in the cognitively healthy elderly population. However, the diagnosis of bvFTD and iNPH was based on the evaluation of a neurologist and a neurosurgeon and there was a consensus on the diagnoses regarding all patients.

\section{Conclusion}

This study provides the first known systematic evidence of the C9ORF72 expansion among iNPH patients. To this end, the current findings strongly support clinical similarity or even potential comorbidity between iNPH and FTLD-ALS spectrum in a subset of patients carrying the C9ORF72 expansion. The clinical symptomatology associated with iNPH and bvFTD appear overlapping, which complicates the differential diagnostics of these two diseases. However, in the current study, the positive shunt response supports the idea of potential comorbidity, rather than unsuccessful differential diagnostics between iNPH and FTLD-ALS spectrum. On the basis of the current evidence, clinicians are encouraged to routinely inquire their patients about the existence of possible behavioral and/or psychiatric problems, together with a family history of ALS/FTLD, to allow for the consistent evaluation of the potential role of FTLD-ALS spectrum in iNPH patients. It is additionally recommended that iNPH patients with cortical atrophy and personality changes, severe neuropsychiatric and/or psychiatric symptoms, would be submitted to an analysis for the potential presence of the C9ORF72 expansion. On the other hand, FTLD patients with enlarged ventricles and severely impaired gait should be evaluated by a neurosurgeon for a potential shunt benefit. Despite the fact that the presence of the $C 9 O R F 72$ expansion in iNPH patients appears quite rare, the frequency of the C9ORF72 expansion in the present iNPH cohort was unexpectedly high. Thus, further investigations into the potential role of the C9ORF72 expansion in other iNPH cohorts are warranted. 
295 Supplementary material

296 No supplementary material.

297 
299 1. Relkin N, Marmarou A, Klinge P, Bergsneider M, Black PM. INPH guidelines, part II:

300 Diagnosing idiopathic normal-pressure hydrocephalus. Neurosurgery. 2005;57(3 SUPPL.):4$301 \quad 16$.

2. Andrén K, Wikkelsø C, Tisell M, Hellström P. Natural course of idiopathic normal pressure hydrocephalus. J Neurol Neurosurg Psychiatry [Internet]. 2014;85(7):806-10. Available from: http://www.ncbi.nlm.nih.gov/pubmed/24292998

3. Malm J, Graff-Radford NR, Ishikawa M, Kristensen B, Leinonen V, Mori E, et al. Influence of comorbidities in idiopathic normal pressure hydrocephalus - research and clinical care. A report of the ISHCSF task force on comorbidities in INPH. Fluids Barriers CNS. 2013;10(1):22.

4. Williams MA, Relkin NR. Diagnosis and management of idiopathic normal-pressure hydrocephalus. Neurol Clin Pract. 2013;3(5):375-85.

5. Saito M, Nishio Y, Kanno S, Uchiyama M, Hayashi A, Takagi M, et al. Cognitive profile of idiopathic normal pressure hydrocephalus. Dement Geriatr Cogn Dis Extra. 2011;1(1):20211.

6. Rascovsky K, Hodges JR, Knopman D, Mendez MF, Kramer JH, Neuhaus J, et al. Sensitivity of revised diagnostic criteria for the behavioural variant of frontotemporal dementia. Brain. 2011;134(9):2456-77.

7. DeJesus-Hernandez M, Mackenzie IR, Boeve BF, Boxer AL, Baker M, Rutherford NJ, et al. Expanded GGGGCC Hexanucleotide Repeat in Noncoding Region of C9ORF72 Causes Chromosome 9p-Linked FTD and ALS. Neuron. 2011;72(2):245-56. 
hexanucleotide repeat expansion in C9ORF72 is the cause of chromosome 9p21-linked ALSFTD. Neuron. 2011;72(2):257-68.

9. Kaivorinne A-L, Bode MK, Paavola L, Tuominen H, Kallio M, Renton AE, et al. Clinical Characteristics of C9ORF72-Linked Frontotemporal Lobar Degeneration. Dement Geriatr Cogn Dis Extra. 2013;3(1):251-62.

10. Hsiung GYR, Dejesus-Hernandez M, Feldman HH, Sengdy P, Bouchard-Kerr P, Dwosh E, et al. Clinical and pathological features of familial frontotemporal dementia caused by C9ORF72 mutation on chromosome 9p. Brain. 2012;135(3):709-22.

11. Korhonen V, Solje E, Suhonen N-M, Rauramaa T, Vanninen R, Remes A, et al. Frontotemporal dementia as a comorbidity to idiopathic normal pressure hydrocephalus (iNPH): A short review of literature and an unusual case. Fluids Barriers CNS. 2017;14(1):10.

12. van der Zee J, Gijselinck I, Dillen L, Van Langenhove T, Theuns J, Engelborghs S, et al. A Pan-European Study of the C9orf72 Repeat Associated with FTLD: Geographic Prevalence, Genomic Instability, and Intermediate Repeats. Hum Mutat. 2013;34(2):363-73.

13. Nuytemans K, Bademci G, Kohli MM, Beecham GW, Wang L, Young JI, et al. C9orf72 intermediate repeat copies are a significant risk factor for parkinson disease. Ann Hum Genet. 2013;77(5):351-63.

14. Theuns J, Verstraeten A, Sleegers K, Wauters E, Gijselinck I, Smolders S, et al. Global investigation and meta-analysis of the C9orf72 (G4C2)n repeat in Parkinson disease. Neurology [Internet]. 2014;83(21):1906-13. Available from: http://www.neurology.org/content/83/21/1906.long

15. Nuytemans K, Inchausti V, Beecham GW, Wang L, Dickson DW, Trojanowski JQ, et al. 
Absence of C9ORF72 expanded or intermediate repeats in autopsy-confirmed Parkinson's disease. Mov Disord. 2014;

16. Schottlaender L V., Polke JM, Ling H, MacDoanld ND, Tucci A, Nanji T, et al. The analysis of C9orf72 repeat expansions in a large series of clinically and pathologically diagnosed cases with atypical parkinsonism. Neurobiol Aging. 2015;36(2):1221.e1-1221.e6.

\section{Cannas A, Solla P, Borghero G, Floris GL, Chio A, Mascia MM, et al. C9ORF72} intermediate repeat expansion in patients affected by atypical parkinsonian syndromes or Parkinson's disease complicated by psychosis or dementia in a Sardinian population. J Neurol [Internet]. 2015;262(11):2498-503. Available from: http://link.springer.com/10.1007/s00415-015-7873-6

18. Wilke C, Pomper JK, Biskup S, Puskás C, Berg D, Synofzik M. Atypical parkinsonism in C9orf72 expansions: a case report and systematic review of 45 cases from the literature. $\mathbf{J}$ Neurol. 2016;263(3):558-74.

19. Lesage S, Le Ber I, Condroyer C, Broussolle E, Gabelle A, Thobois S, et al. C9orf72 repeat expansions are a rare genetic cause of parkinsonism. Brain. 2013;136(2):385-91.

20. Cavallieri F, Mandrioli J, Rosafio F, Contardi S, Fasano A, Menozzi E, et al. C9ORF72 and parkinsonism: Weak link, innocent bystander, or central player in neurodegeneration? J Neurol Sci. 2017;378:49-51.

21. Espay AJ, Da Prat GA, Dwivedi AK, Rodriguez-Porcel F, Vaughan JE, Rosso M, et al. Deconstructing normal pressure hydrocephalus: Ventriculomegaly as early sign of neurodegeneration. Vol. 82, Annals of Neurology. 2017. p. 503-13.

22. Harris JM, Gall C, Thompson JC, Richardson AMT, Neary D, Du Plessis D, et al. Sensitivity and specificity of FTDC criteria for behavioral variant frontotemporal dementia. Neurology. 
23. McGirt MJ, Woodworth G, Coon AL, Thomas G, Williams MA, Rigamonti D. Diagnosis, Treatment, and Analysis of Long-term Outcomes in Idiopathic Normal-Pressure Hydrocephalus. Neurosurgery. 2005;57(4):699-705.

24. Thomas G, McGirt MJ, Woodworth GF, Heidler J, Rigamonti D, Hillis AE, et al. Baseline neuropsychological profile and cognitive response to cerebrospinal fluid shunting for idiopathic normal pressure hydrocephalus. Dement Geriatr Cogn Disord. 2005;20(2-3):1638.

25. Toma AK, Papadopoulos MC, Stapleton S, Kitchen ND, Watkins LD. Systematic review of the outcome of shunt surgery in idiopathic normal-pressure hydrocephalus. In: Acta Neurochirurgica. 2013. p. 1977-80.

26. Klinge P, Hellström P, Tans J, Wikkels $\emptyset$ C. One-year outcome in the European multicentre study on iNPH. Acta Neurol Scand. 2012;126(3):145-53.

27. Mackenzie IRA, Frick P, Neumann M. The neuropathology associated with repeat expansions in the C9ORF72 gene. Acta Neuropathol. 2014;127(3):347-57.

28. Yokoyama JS, Sirkis DW, Miller BL. C9ORF72 hexanucleotide repeats in behavioral and motor neuron disease: clinical heterogeneity and pathological diversity. Am J Neurodegener Dis. $2014 ; 3(1): 1-18$.

29. Bieniek KF, Murray ME, Rutherford NJ, Castanedes-Casey M, Dejesus-Hernandez M, Liesinger AM, et al. Tau pathology in frontotemporal lobar degeneration with C9ORF72 hexanucleotide repeat expansion. Acta Neuropathol. 2013;125(2):289-302.

30. Kamalainen A, Herukka SK, Hartikainen P, Helisalmi S, Moilanen V, Knuuttila A, et al. Cerebrospinal fluid biomarkers for Alzheimer's disease in patients with frontotemporal lobar 
degeneration and amyotrophic lateral sclerosis with the C9ORF72 repeat expansion. Dement Geriatr Cogn Disord. 2015;39(5-6):287-93.

31. Leinonen V, Koivisto AM, Savolainen S, Rummukainen J, Sutela A, Vanninen R, et al. Postmortem findings in 10 patients with presumed normal-pressure hydrocephalus and review of the literature. Neuropathol Appl Neurobiol. 2012;38(1):72-86.

32. Vanhala V, Junkkari A, Korhonen VE, Kurki MI, Hiltunen M, Rauramaa T, et al. Prevalence of Schizophrenia in Idiopathic Normal Pressure Hydrocephalus. Neurosurgery. 2018;0(0):17.

33. Mahoney CJ, Downey LE, Ridgway GR, Beck J, Clegg S, Blair M, et al. Longitudinal neuroimaging and neuropsychological profiles of frontotemporal dementia with C9ORF72 expansions. Alzheimers Res Ther. 2012;4(5):41.

34. Kato T, Sato H, Emi M, Seino T, Arawaka S, Iseki C, et al. Segmental copy number loss of SFMBT1 gene in elderly individuals with ventriculomegaly: a community-based study. Intern Med. 2011;50(4):297-303.

35. Portenoy RK, Berger A, Gross E. Familial occurrence of idiopathic normal-pressure hydrocephalus. Arch Neurol. 1984;41(3):335-7.

36. Huovinen J, Kastinen S, Komulainen S, Oinas M, Avellan C, Frantzen J, et al. Familial idiopathic normal pressure hydrocephalus. J Neurol Sci. 2016;368:11-8.

37. Takahashi Y, Kawanami T, Nagasawa H, Iseki C, Hanyu H, Kato T. Familial normal pressure hydrocephalus (NPH) with an autosomal-dominant inheritance: A novel subgroup of NPH. J Neurol Sci. 2011;308(1-2):149-51.

38. Cusimano MD, Rewilak D, Stuss DT, Barrera-Martinez JC, Salehi F, Freedman M. Normalpressure hydrocephalus: is there a genetic predisposition? Can J Neurol Sci. 2011;38(2):274- 
81.

414 39. Korhonen VE, Helisalmi S, Jokinen A, Jokinen I. Copy number loss in SFMBT1 is common among Finnish and Norwegian patients with iNPH. 2018;(1):1-8.

40. Lee L. Riding the wave of ependymal cilia: Genetic susceptibility to hydrocephalus in primary ciliary dyskinesia. J Neurosci Res. 2013;91(9):1117-32.

Fig 1: Flowchart

Fig 2: Neuroradiology enlarged ventricles with Evan's index of 0.36-0.50. Evident frontotemporal (g, same patient as in b), and frontoparietal cortical atrophy (h, same patient as in $d)$.

p62 immunopositivity $(A, B)$, thin TDP-43 immunoreactive neuritis $(C)$ and moderate amount of $A \beta$ immureactive plaques (D). Arrowheads marking example neuropathological changes.

430 Table 1: Demographics of the study cohort and controls

431 Table 2: Clinical characteristics and pathological findings of the iNPH patients with the C9ORF72 432 expansion

433 Table 3: Neuroradiological features of the C9ORF72 expansion carriers 
434 Table 4: Cognitive profile of the C9ORF72 expansion carriers

435 